\title{
Prognosis of patients undergoing emergency surgery for type A acute aortic dissection without exclusion of the intimal tear
}

\author{
Satoshi Unosawa, MD, PhD, ${ }^{\text {a }}$ Mitsumasa Hata, $\mathrm{MD}, \mathrm{PhD},{ }^{\mathrm{b}}$ Tetsuya Niino, $\mathrm{MD}, \mathrm{PhD},{ }^{\mathrm{a}}$ \\ Kazuma Shimura, MD, $\mathrm{PhD},{ }^{\mathrm{a}}$ and Motomi Shiono, $\mathrm{MD}, \mathrm{PhD}^{\mathrm{b}}$
}

\begin{abstract}
Objectives: To investigate the prognosis after emergency surgery for acute type A aortic dissection with an unknown entry site and to identify the predictors of postoperative aortic dilatation.
\end{abstract}

\begin{abstract}
Methods: The subjects were 102 patients undergoing emergency surgery for acute type A dissection from July 2005 to October 2010. They were divided into group $I(n=45)$ undergoing aortic surgery without tear resection and group II $(\mathrm{n}=57)$ undergoing resection that included the intimal tear.

Results: The postoperative hospital mortality was similar, $13.3 \%(n=6)$ in group I and $12.3 \%(n=7)$ in group II. Of the 102 patients, 69 underwent follow-up computed tomography scanning after discharge, and the aortic diameter was significantly increased in group I compared with that in group II $(P=.035)$. Dilatation of the descending aorta occurred in 21 patients $(30.4 \%)$. Multivariate logistic regression analysis revealed that a patent false lumen $(P=.027)$ and nonexclusion of the entry site $(P=.012)$ were independent risk factors for aortic dilatation. No difference was found in the freedom from aorta-related clinical events at 4 years, with a rate of $81.9 \%$ in group I and $74.4 \%$ in group II. Also, no difference was found in the 4-year actuarial survival rate between groups I and II $(86.4 \%$ and $78.5 \%$, respectively).
\end{abstract}

Conclusions: The prognosis of patients without exclusion of the entry site was acceptable. Careful follow-up is needed for patients with a patent false lumen or nonexcluded entry because of the risk of aortic dilatation. (J Thorac Cardiovasc Surg 2013;146:67-71)

The management of acute type A aortic dissection (AAAD) is problematic, and it is still unclear how extensive the aortic resection should be for patients with this life-threatening condition. Our fundamental strategy for AAAD involves excision of the intimal tear. In most patients, ascending aortic or proximal arch replacement is sufficient because the intimal tear is generally located in the concavity of the transverse part of the arch. ${ }^{1}$ When the tear is located in the aortic arch, however, total arch replacement should be performed to achieve tear excision. If the intimal tear is not identified anywhere from the ascending aorta to the distal arch, our treatment strategy has been to simply replace the proximal arch and conduct careful follow-up after surgery. However, a risk exists of the residual aorta expanding because the intimal tear has not been resected. In the present study, we reviewed the data from patients undergoing aortic resection for AAAD without tear excision and assessed their medium-term outcomes.

\footnotetext{
From the Department of Cardiovascular Surgery, ${ }^{a}$ Disaster Medical Center, Tokyo, Japan; and Department of Cardiovascular Surgery, ${ }^{\mathrm{b}}$ Nihon University School of Medicine, Tokyo, Japan.

Disclosures: Authors have nothing to disclose with regard to commercial support. Received for publication Jan 28, 2012; revisions received April 18, 2012; accepted for publication May 17, 2012; available ahead of print June 22, 2012.

Address for reprints: Satoshi Unosawa, MD, PhD, Department of Cardiovascular Surgery, Disaster Medical Center, 3256, Midori-chou, Tachikawa-shi, Tokyo 190-0014, Japan (E-mail: unosaw@gmail.com).

$0022-5223 / \$ 36.00$

Copyright (c) 2013 by The American Association for Thoracic Surgery doi:10.1016/j.jtcvs.2012.05.067
}

\section{METHODS}

From July 2005 to October 2010, 116 patients with AAAD underwent emergency surgery at our institutions. Contrast computed tomography (CT) was performed in all patients as soon as they were referred to the hospital. This was followed by transthoracic echocardiography to detect pericardial effusion and to assess for aortic regurgitation and cardiac function. Even if the initial CT scan revealed a thrombosed false lumen, we recommended emergency surgery. Patients were transferred to the operating room as soon as possible after informed consent to surgery was obtained. Of the 116 patients, 7 who had undergone total arch replacement and 7 with Debakey type II aortic dissection were excluded from the present study.

In 45 patients, the intimal tear was not located anywhere from the ascending aorta to the distal arch; thus, aortic resection did not include the tear (group I). The remaining 57 patients, with the entry site located in the ascending aorta or proximal aortic arch, underwent resection that included the tear and ascending aortic or hemiarch replacement (group II).

The institutional review board approved the reporting of the information obtained from our retrospective study.

\section{Surgical Procedure}

We previously reported the surgical technique. ${ }^{2-5}$ Cardiopulmonary bypass was implemented after cannulation of the femoral artery. A 2-stage venous cannula was inserted into the right atrium. The ascending aorta was then opened longitudinally under moderate hypothermic arrest $\left(28^{\circ} \mathrm{C}\right)$ without cerebral perfusion. Replacement of the ascending aorta or hemiarch replacement was performed with a Dacron graft after both the distal and the proximal stumps of the aorta were reinforced with Teflon felt and gelatin resorcin formalin glue. Antegrade systemic circulation was re-established through a side branch of the graft after completion of the open distal anastomosis.

\section{CT Follow-up}

Enhanced CT scanning was performed at our outpatient clinic 1 month after surgery, and CT was repeated annually thereafter. The maximum 


\section{Abbreviations and Acronyms}

$\mathrm{AAAD}=$ acute type A aortic dissection

$\mathrm{CT}=$ computed tomography

diameter of the residual thoracic aorta and the patency of the false lumen were assessed on the CT scans. On the final scan, the aortic diameter was measured at the level at which it was largest on the first postoperative scan. An aortic diameter of $60 \mathrm{~mm}$ or larger was defined as an aneurysmal change. Dilatation of the descending aorta was defined as an increase in the maximal diameter by $5 \mathrm{~mm}$ or more during the follow-up period. Aortarelated clinical events were defined as aneurysmal change, reoperation, and death.

Operative mortality was defined as any death within 30 days after surgery in or outside the hospital. In-hospital mortality was defined as any death that occurred before discharge from our hospital or another hospital to which the patient had been transferred.

\section{Statistical Analysis}

Continuous variables are reported as the mean \pm standard deviation. For comparison of the 2 groups, Student $t$ test was used for continuous variables and the chi-square test for categorical variables. For multivariate analysis, only variables with $P<.01$ on univariate analysis were entered into the logistic regression model. The Kaplan-Meier method was used to determine the survival rate and the freedom from aorta-related clinical event rate. Statistical analyses were performed using SPSS software, version 11 (SPSS, Chicago, Ill).

\section{RESULTS}

The clinical profile and dissection characteristics of the 2 groups are listed in Table 1. Compared with group I, group II had significantly greater rates of moderate to severe aortic regurgitation $(P=.016)$ and a patent false lumen in the ascending aorta $(P=.015)$. Group II patients were also significantly older than those in group I (Table 1 ). The average duration of cardiopulmonary bypass, aortic crossclamp time, and circulatory arrest time were all significantly shorter in group I than in group II. A modified Bentall procedure was performed in 3 patients with severe aortic regurgitation because of marked dilatation of the aortic root. One patient underwent separate aortic valve replacement for a bicuspid valve. No differences were found in the incidence of postoperative neurologic deficits, pneumonia, deep sternal wound infection, or renal failure between the 2 groups. The postoperative hospital mortality was also similar at $13.3 \%$ (6 patients) in group I and $12.3 \%$ (7 patients) in group II (Table 2).

Of the 89 survivors, 2 died within 5 months after discharge from the hospital of cerebral infarction and rupture of the thoracic aortic aneurysm, respectively. Eight of the survivors did not undergo enhanced CT scanning owing to renal insufficiency, contrast medium allergy, or other reasons. Ten survivors were referred to other hospitals for ongoing treatment. Sixty-nine patients underwent follow-up CT scanning after discharge. The interval between surgery and the final CT scan was 12 to 59 months (mean, $33.0 \pm$ 13.4). On the initial postoperative CT scans, the incidence of a patent false lumen and the aortic diameter was similar in the 2 groups. However, the extent of aortic dilatation over time was significantly greater in group I than in group II (Table 3).

Dilatation of the descending aorta occurred in 21 patients $(30.4 \%)$, and the site of dilatation was the distal arch in 12. Multivariate analysis revealed that a patent false lumen (odds ratio, 3.91; 95\% confidence interval, $1.17-13.16 ; P=.027$ ) and excision of the tear (odds ratio, $0.21 ; 95 \%$ confidence interval, $0.06-0.71 ; P=.012)$ were significant predictors of dilatation of the descending aorta. Two patients in each group developed an aneurysmal change of the residual aorta. In group I, 1 patient required total arch replacement because of rapid expansion of the aorta from $49 \mathrm{~mm}$ to $67 \mathrm{~mm}$ within 3 months, and 1 underwent descending aortic replacement 3 years after the initial operation. In group II, 1 patient underwent replacement of the descending aorta 1 year after the initial operation, and 1 refused surgery for an aneurysm with a diameter of $61 \mathrm{~mm}$. In group I, 1 patient died of sepsis 32 months after surgery; however, no late deaths occurred in group II.

No difference was found in the freedom from aortarelated clinical events at 4 years $(81.9 \%$ in group I and $74.4 \%$ in group II; Figure 1). Also, no difference was found in the 4-year actuarial survival rate $(86.4 \%$ in group I and $78.5 \%$ in group II; Figure 2).

\section{DISCUSSION}

According to our management protocol, the chief priorities of emergency surgery for AAAD are primary excision of the intimal tear and avoidance of serious complications. Several investigators have advocated routine extended or total aortic arch resection for the initial surgical management of AAAD, irrespective of the location of the intimal tear. ${ }^{6,7}$ Although they have achieved satisfactory results, it is important to remember that $\mathrm{AAAD}$ is a lethal condition, and the first priority is to ensure that the patient lives. If the acute event is not fatal, this constitutes success, regardless of possible later aortic problems. Extended resection will necessarily increase the alreadyhigh operative risk. Ehrlich and colleagues ${ }^{8}$ reported that the site of the intimal tear did not influence the outcome, although mortality was greater when the resection was more extensive. Bachet and colleagues ${ }^{9}$ reported that closure of the entry site at the initial emergency operation led to a lower reoperation rate. Westaby and colleagues ${ }^{10}$ also advocated the policy of primary tear excision (ie, the "conservative pathology-oriented approach"). The only concern is that it remains unclear how extensive the resection should be if no intimal tear can be identified from the ascending aorta to the aortic arch. 
TABLE 1. Clinical profile and dissection characteristics

\begin{tabular}{|c|c|c|c|}
\hline Characteristics & $\begin{array}{l}\text { Group I } \\
(n=45)\end{array}$ & $\begin{array}{c}\text { Group II } \\
(\mathbf{n}=\mathbf{5 7})\end{array}$ & $\begin{array}{c}P \\
\text { value } \\
\end{array}$ \\
\hline \multicolumn{4}{|l|}{ Clinical } \\
\hline Age (y) & $61.1 \pm 12.8$ & $66.7 \pm 13.5$ & .035 \\
\hline Male gender & $30(66.7)$ & $28(49.1)$ & .076 \\
\hline Hypertension & $31(68.9)$ & $40(70.2)$ & .888 \\
\hline Smoking & $4(8.9)$ & $3(5.3)$ & .472 \\
\hline Diabetes & 0 & $1(1.8)$ & .372 \\
\hline Dyslipidemia & $6(13.3)$ & $5(8.8)$ & 461 \\
\hline Marfan syndrome & 0 & $3(5.3)$ & .118 \\
\hline CVD & $3(6.7)$ & $6(10.5)$ & .495 \\
\hline IHD & $3(6.7)$ & $5(8.8)$ & .695 \\
\hline COPD & $1(2.2)$ & $3(5.3)$ & .703 \\
\hline Renal failure & $4(8.9)$ & $4(7.0)$ & .727 \\
\hline \multicolumn{4}{|l|}{ Dissection } \\
\hline False lumen thrombosis & $25(5.6)$ & 18 (31.6) & .015 \\
\hline Shock & $11(24.4)$ & $24(42.1)$ & .062 \\
\hline Intubation & $3(6.7)$ & $6(10.5)$ & .495 \\
\hline CPR & 0 & $3(5.3)$ & .118 \\
\hline Moderate to severe AR & $3(6.7)$ & $14(24.6)$ & .016 \\
\hline \multicolumn{4}{|l|}{ Malperfusion } \\
\hline Peripheral & $4(8.9)$ & $5(8.8)$ & .984 \\
\hline Cerebral & $2(4.4)$ & $1(1.8)$ & .425 \\
\hline Cardiac & $2(4.4)$ & $5(8.8)$ & .391 \\
\hline
\end{tabular}

$A R$, Aortic regurgitation; $C O P D$, chronic obstructive pulmonary disease; $C P R$, cardiopulmonary resuscitation; $C V D$, cerebrovascular disease; $I H D$, ischemic heart disease

Fundamentally, the aim of emergency surgery for AAAD is to prevent subsequent complications, such as myocardial infarction, acute aortic regurgitation, and cardiac tamponade, or to relieve existing complications associated with the risk of sudden death. An emergency procedure that involves opening the pericardium and replacement of the ascending aorta or hemiarch replacement is enough to salvage the patient, even if the intimal tear remains in the distal arch or descending aorta. In the present study, the hospital and late mortality rates of both groups were low, and aortic resection for AAAD without excision of the intimal tear was not associated with an increased incidence of reoperation, although nonexclusion of the entry site was an independent risk factor for late aortic dilatation.

Postoperative patency and dilatation of the false lumen are also important issues, which has been emphasized recently by several reports. ${ }^{11-15}$ Kimura and colleagues ${ }^{16}$ reported that the patent false lumen influenced postoperative aortic enlargement. In the present study, the false lumen was patent in $53.6 \%$ of all patients, and no difference was found in the patency rate between the 2 groups. This was considered to be acceptable with reference to previous reports. David and colleagues ${ }^{17}$ reported that the prevalence of a patent false lumen after surgery was reduced from $91 \%$ to $59 \%$ with the open distal anastomosis technique. We believe that emergency open repair and fixation of the distal aortic stump with gelatin resorcin formalin glue decreases
TABLE 2. Surgical data and early outcome

\begin{tabular}{|c|c|c|c|}
\hline Variable & $\begin{array}{l}\text { Group I } \\
(n=45)\end{array}$ & $\begin{array}{l}\text { Group II } \\
(\mathbf{n}=\mathbf{5 7})\end{array}$ & $\begin{array}{c}P \\
\text { value }\end{array}$ \\
\hline Replacement procedure & & & $<.001$ \\
\hline AAR & 41 & 33 & \\
\hline HAR & 4 & 24 & \\
\hline CABG & 0 & $4(7.0)$ & .070 \\
\hline Modified Bentall procedure & 0 & $3(5.3)$ & .118 \\
\hline AVR & 0 & $1(1.8)$ & .372 \\
\hline CPBT (min) & $95.5 \pm 26.7$ & $121 \pm 39.2$ & $<.001$ \\
\hline $\mathrm{ACCT}(\min )$ & $54.8 \pm 12.6$ & $70.7 \pm 22.5$ & $<.001$ \\
\hline CAT (min) & $21.0 \pm 5.4$ & $26.6 \pm 10.3$ & .001 \\
\hline Lowest rectal temperature $\left({ }^{\circ} \mathrm{C}\right)$ & $26.6 \pm 1.6$ & $25.7 \pm 2.4$ & .025 \\
\hline \multicolumn{4}{|l|}{ Complications } \\
\hline Neurologic deficits & $3(6.7)$ & $5(8.8)$ & .695 \\
\hline Pneumonia & $3(6.7)$ & $5(8.8)$ & .695 \\
\hline Renal failure & $1(2.2)$ & $2(3.5)$ & .703 \\
\hline Mediastinitis & 0 & $1(1.8)$ & .372 \\
\hline $\begin{array}{l}\text { Prolonged mechanical ventilation } \\
\quad(>24 \mathrm{~h})\end{array}$ & $10(22.2)$ & $19(33.3)$ & .217 \\
\hline Hospital stay (d) & $28.3 \pm 62.6$ & $21.1 \pm 23.2$ & .422 \\
\hline Operative mortality & $3(6.7)$ & $6(10.5)$ & .495 \\
\hline In-hospital mortality & $6(13.3)$ & $7(12.3)$ & .874 \\
\hline
\end{tabular}

the patent false lumen rate and possibly improves the long-term survival of patients with AAAD. ${ }^{18}$ No difference was found in the freedom from reoperation on the distal aorta between our 2 groups. Therefore, we consider it is sufficient to repair type A dissection to a level at which it corresponds to type $\mathrm{B}$ dissection, even if the intimal tear is not located in the distal aortic arch. It has been reported that the 3 -year mortality rate of patients with type B dissection (even with a patent false lumen) is only $10 \%$ to $20 \% .^{19,20}$ We have also reported that the long-term survival rate at 5 and 10 years for patients with type B dissection receiving medical treatment was $89.4 \%$ and $71.8 \%$, respectively, regardless of their false lumen status. ${ }^{21}$ Therefore, careful observation and management of hypertension are essential for maintaining event-free survival in patients with AAAD in

TABLE 3. Computed tomography findings

\begin{tabular}{lccc}
\hline \multicolumn{1}{c}{ Finding } & $\begin{array}{c}\text { Group I } \\
(\mathbf{n = 3 5})\end{array}$ & $\begin{array}{c}\text { Group II } \\
(\mathbf{n = 3 4 )}\end{array}$ & $\begin{array}{c}\boldsymbol{P} \\
\text { value }\end{array}$ \\
\hline Aortic diameter & & & \\
$\quad$ Initial postoperative CT scan & $39.9 \pm 5.9$ & $39.2 \pm 6.7$ & .617 \\
$\quad$ Latest CT scan & $44.1 \pm 8.7$ & $40.6 \pm 8.6$ & .091 \\
Aortic dilatation & $3.99 \pm 5.3$ & $1.44 \pm 4.5$ & .035 \\
Aortic dilatation > 5 mm & $16(45.7)$ & $5(14.7)$ & .005 \\
Maximal aortic diameter $>50 \mathrm{~mm}$ & $9(25.7)$ & $5(14.7)$ & .256 \\
False lumen thrombosis & $14(40.0)$ & $18(52.9)$ & .281 \\
\hline
\end{tabular}

CT, Computed tomography. 


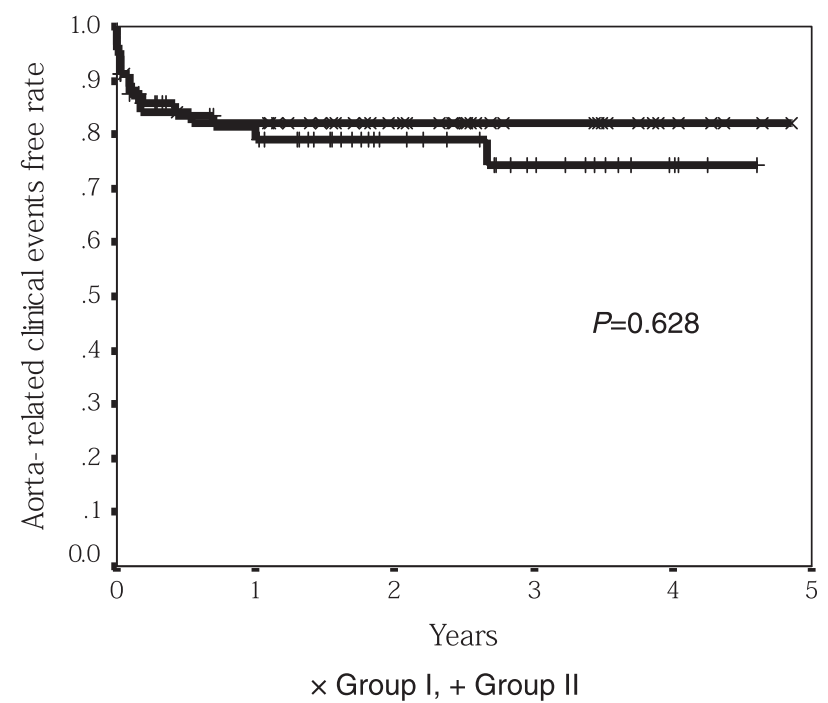

FIGURE 1. Freedom from aorta-related clinical events.

the long term, regardless of whether entry resection has been achieved by the initial emergency surgery.

\section{Study Limitations}

The present study had several limitations, including a relatively small number of subjects and a short follow-up period. Moreover, only $69(77.5 \%)$ of the 89 patients discharged from the hospital underwent follow-up CT scanning. In particular, a greater number of patients were lost from group II, and this might have affected the findings from the follow-up CT scanning. No differences were found in the freedom from aorta-related clinical events and survival between the 2 groups, although the aortic diameter of the patients without exclusion of the intimal tear showed

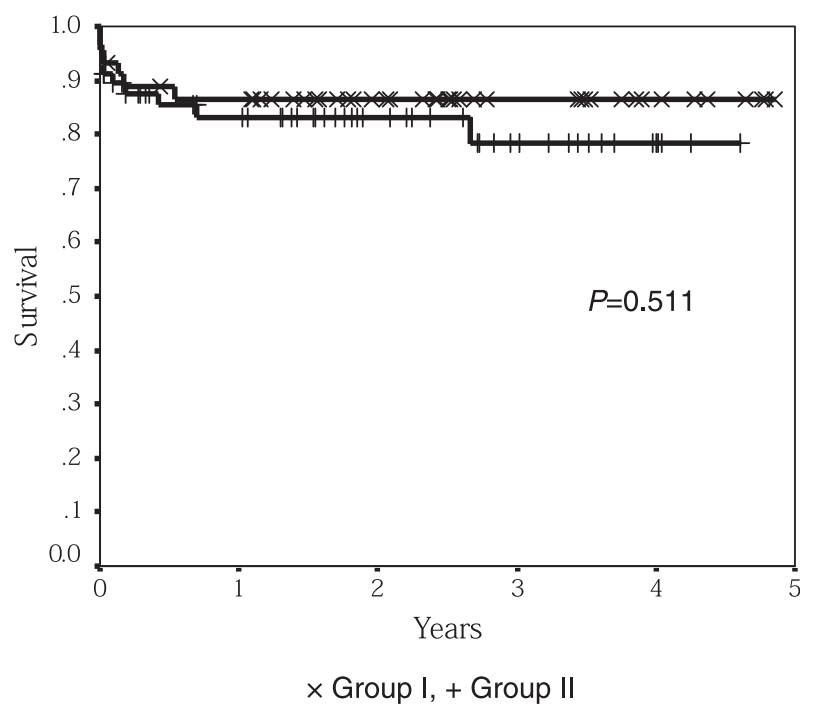

FIGURE 2. Actuarial survival. significantly more expansion after medium-term follow-up. An assessment of a larger number of patients for a longer follow-up period might have affected the freedom from aorta-related events or survival. Therefore, prospective studies on a larger scale with a longer follow-up period are required.

\section{CONCLUSIONS}

We assessed the medium-term outcomes of surgery for AAAD with an unknown site of entry. The prognosis of patients without exclusion of the intimal tear was acceptable. However, it is important to perform careful follow-up, because a patent false lumen and nonexclusion of the entry are risk factors for aortic dilatation.

\section{References}

1. Bachet JE. Acute type A aortic dissection: can we dramatically reduce the surgical mortality? Ann Thorac Surg. 2002;73:701-3.

2. Hata M, Suzuki M, Sezai A, Niino T, Unosawa S, Minami K, et al. Less invasive quick replacement for octogenarians with type A acute aortic dissection. $J$ Thorac Cardiovasc Surg. 2008;136:489-93.

3. Hata M, Sezai A, Yoshitake I, Wakui S, Minami K, Shiono M. Midterm outcomes of rapid, minimally invasive resection of acute type A aortic dissection in octogenarians. Ann Thorac Surg. 2010;89:1860-4.

4. Hata M, Suzuki M, Sezai A, Niino T, Yoshitake I, Unosawa S, et al. Less invasive quick replacement of the proximal arch with aggressive rapid rewarming for type A acute aortic dissection. Surg Today. 2009;39:353-5.

5. Hata M, Suzuki M, Sezai A, Niino T, Yoshitake I, Unosawa S, et al. Outcome of less invasive proximal arch replacement with moderate hypothermic circulatory arrest followed by aggressive rapid re-warming in emergency surgery for type A acute aortic dissection. Circ J. 2009;73:69-72.

6. Kazui T, Washiyama N, Muhammad BA, Terada H, Yamashita K, Takinami M, et al. Extended total arch replacement for acute type A aortic dissection: experience with seventy patients. J Thorac Cardiovasc Surg. 2000;119:558-65.

7. Hirotani T, Kameda T, Kumamoto T, Shirota S. Results of total aortic arch replacement for an acute aortic arch dissection. J Thorac Cardiovasc Surg. 2000;120:686-91.

8. Ehrlich MP, Ergin MA, McCullough JN, Lansman SL, Galla JD, Griepp RB, et al. Results of immediate surgical treatment of all acute type A dissection. Circulation. 2000;102:III248-52.

9. Bachet JE, Termignon JL, Dreyfus G, Goudot B, Martinelli L, Brodaty D, et al. Aortic dissection: prevalence, cause, and results of late reoperations. J Thorac Cardiovasc Surg. 1994;108:199-205.

10. Westaby S, Saito S, Katsumata T. Acute type A dissection: conservative methods provide consistently low mortality. Ann Thorac Surg. 2002;73:707-13.

11. Bernard Y, Zimmermann H, Chocron S, Litzler JF, Kastler B, Etievent JP, et al. False lumen patency as a predictor of late outcome in aortic dissection. Am J Cardiol. 2001;87:1378-82.

12. Moore NR, Parry AJ, Trottman-Dickenson B, Pillai R, Westaby S. Fate of the native aorta after repair of acute type A dissection: a magnetic resonance imaging study. Heart. 1996;75:62-6.

13. Fattori R, Bacchi-Reggiani L, Bertaccini P, Napoli G, Fusco F, Longo M, et al. Evolution of aortic dissection after surgical repair. Am J Cardiol. 2000;86: 868-72.

14. Fattouch K, Sampognaro R, Navarra E, Caruso M, Pisano C, Coppola G, et al. Long-term results after repair of type A acute aortic dissection according to false lumen patency. Ann Thorac Surg. 2009;88:1244-50.

15. Halstead JC, Meier M, Etz C, Spielvogel D, Bodian C, Wurm M, et al. The fate of the distal aorta after repair of acute type A aortic dissection. J Thorac Cardiovasc Surg. 2007;133:127-35.

16. Kimura N, Tanaka M, Kawahito K, Yamaguchi A, Ino T, Adachi H. Influence of patent false lumen on long-term outcome after surgery for acute type A aortic dissection. J Thorac Cardiovasc Surg. 2008;136:1160-6.

17. David TE, Armstrong S, Ivanov J, Barnard S. Surgery for acute type A aortic dissection. Ann Thorac Surg. 1999;67:1999-2001. 
18. Hata M, Shiono M, Sezai A, Iida M, Negishi N, Sezai A. Type A acute aortic dissection: immediate and mid-term results of emergency aortic replacement with the aid of gelatin resorcin formalin glue. Ann Thorac Surg. 2004;78:853-7.

19. Tsai TT, Evangelista A, Nienaber CA, Myrmel T, Meinhardt G, Cooper JV, et al Partial thrombosis of the false lumen in patients with acute type B aortic dissection. N Engl J Med. 2007;357:349-59.
20. Akutsu K, Nejima J, Kiuchi K, Sasaki K, Ochi M, Tanaka K, et al. Effect of the patent false lumen on the long-term outcome of the type B acute aortic dissection. Eur J Cardiothorac Surg. 2004;26:359-66.

21. Hata M, Sezai A, Niino T, Wakui S, Unosawa S, Minami K, et al. Prognosis for patients with type B acute aortic dissection: risk analysis of early death and requirement for elective surgery. Circ J. 2007;71:1279-82.

Access to The Journal of Thoracic and Cardiovascular Surgery Online is reserved for print subscribers!

Full-text access to The Journal of Thoracic and Cardiovascular Surgery Online is available for all print subscribers. To activate your individual online subscription, please visit The Journal of Thoracic and Cardiovascular Surgery Online, point your browser to http://www.mosby.com/jtcvs, follow the prompts to activate your online access, and follow the instructions. To activate your account, you will need your subscriber account number, which you can find on your mailing label (note: the number of digits in your subscriber account number varies from 6 to 10 ). See the example below in which the subscriber account number has been circled:

\section{Sample mailing label}

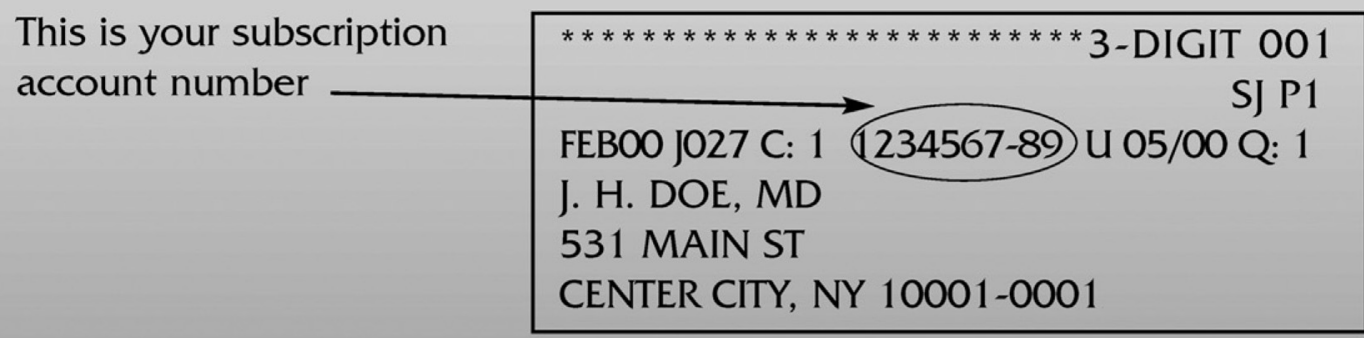

Personal subscriptions to The Journal of Thoracic and Cardiovascular Surgery Online are for individual use only and may not be transferred. Use of The Journal of Thoracic and Cardiovascular Surgery Online is subject to agreement to the terms and conditions as indicated online. 